INPLASY

PROTOCOL

To cite: Wang et al.

Moxibustion for stable chronic obstructive pulmonary

disease: a protocol for

systematic review and meta-

analysis. Inplasy protocol

202140047. doi:

10.37766/inplasy2021.4.0047

Received: 08 April 2021

Published: 08 April 2021

Corresponding author:

Yanping Wang

wangyp1119@outlook.com

Author Affiliation:

Postgraduate Education, Jiangxi University of

Traditional Chinese Medicine

Support: GJJ190657 and

JXSYLXK-ZHYAO148.

Review Stage at time of this submission: The review has not yet started.

Conflicts of interest:

None declared.

\section{Moxibustion for stable chronic obstructive pulmonary disease: a protocol for systematic review and meta-analysis}

Wang, Y1; Huang, M2; Tang, L3; Xu, L4; Wu, J5; Wang, F6; Zhang, $\mathrm{Y}^{7}$.

Review question / Objective: Can moxibustion treat chronic obstructive pulmonary disease in stable period effectively alleviate or prevent lung function and improve the quality of life of patients?

Condition being studied: Patients with stable chronic obstructive pulmonary disease.

Information sources: We will systematically search seven databases (PubMed, EMBASE, CENTRAL, China Biomedical Literature Database, Chinese National Knowledge Infrastructure, Wanfang Data, and VIP) and two clinical trial registration platforms (Clinicaltrials.gov, Chinese Clinical Trial Registry). Reference lists of relevant reviews will also search for adding relevant studies.

INPLASY registration number: This protocol was registered with the International Platform of Registered Systematic Review and Meta-Analysis Protocols (INPLASY) on 08 April 2021 and was last updated on 08 April 2021 (registration number INPLASY202140047).

\section{INTRODUCTION}

Review question / Objective: Can moxibustion treat chronic obstructive pulmonary disease in stable period effectively alleviate or prevent lung function and improve the quality of life of patients?
Rationale: Chronic obstructive pulmonary disease (COPD)is a common chronic respiratory disease, and the main clinical manifestations are dyspnea, cough and/or sputum expectoration. The prevalence and mortality of COPD are both high, causing serious harm to human health and a heavy economic burden on patients, their families 
and society. Although western medicine can relieve the suffering of patients, it is highly dependent. As one of the important components of non-drug therapy, moxibustion has attracted much attention due to its unique treatment methods and curative advantages, and is widely used in chronic respiratory diseases. At present, a randomized controlled trial (RCT) of moxibustion for the treatment of patients with stable COPD has been carried out. The results suggest that moxibustion can stimulate the body's meridians, increase lung capacity, and improve the ventilation function of patients with COPD. The moxa smoke in moxibustion has anti-oxidation and inhibits inflammatory cytokines, thereby improving human immunity. Therefore, this study aims to explore the efficacy and safety of moxibustion in the treatment of COPD, and to provide highquality clinical evidence.

Condition being studied: Patients with stable chronic obstructive pulmonary disease.

\section{METHODS}

Search strategy: Search strategy in PubMed is: (chronic obstructive pulmonary disease [mh] OR pulmonary disease[tw] OR chronic obstructive[tw] OR chronic obstructive airway disease[tw] OR airflow obstructions[tw] OR chronic airflow obstruction[tw] OR emphysema[tw] OR pulmonary emphysema[tw] OR respiratory insufficienty[tw]) AND (moxibustion[mh] OR moxibustion[tw] OR moxa[tw]) NOT (animals[mh] NOT humans[mh]).

Participant or population: Patients with stable chronic obstructive pulmonary disease.

Intervention: The intervention measures will be moxibustion combined with conventional western medicine treatment or moxibustion alone. Moxibustion combined with other traditional chinese medicine treatments (e.g., acupoint application, cupping, massage, acupuncture, etc.) will be excluded.
Comparator: The comparator is conventional western medicine alone, such as bronchodilators, glucocorticoids, mucolytic agents, antioxidants and immunomodulators.

Study designs to be included: Randomized controlled trials.

Eligibility criteria: Inclusion criteria: patients definitely diagnose as a patient with stable COPD. Exclusion criteria: We will exclude the acute phase of COPD, or combine with other complications: such as hypertension, diabetes, coronary heart disease, heart failure.

Information sources: We will systematically search seven databases (PubMed, EMBASE, CENTRAL, China Biomedical Literature Database, Chinese National Knowledge Infrastructure, Wanfang Data, and VIP) and two clinical trial registration platforms (Clinicaltrials.gov, Chinese Clinical Trial Registry). Reference lists of relevant reviews will also search for adding relevant studies.

Main outcome(s): Forced expiratory volume in one second/ forced vital capacity.

Additional outcome(s): 1. Forced expiratory volume in one second; 2 . Forced vital capacity; 3. Maximum volume; 4. Six minutes walking distance; 5. COPD assessment test score; 6. Response to treatment according to recognized classification criteria, such as Guiding Principles for Clinical Research of New Chinese Medicines; 7. Adverse events.

Data management: Two reviewers will independently and repeatedly screen the literature and extract data according to the inclusion/exclusion criteria. First, the obvious irrelevant literature will be excluded by the reading title and abstract. The remaining documents read the full text again to determine the studies that meet all the inclusion criteria. Data extraction includes: (i) study characteristics: first author, publication time, disease inclusion and exclusion criteria, sample size, intervention measures; (ii) Patient 
characteristics: age, gender, course of disease, treatment course; (iii) Outcomes: the continuous outcome extracts the mean and standard deviation/standard error of each group.

Quality assessment / Risk of bias analysis: The Cochrane Handbook tool will be used to evaluate the risk of bias for inclusion in the RCT. Items included: whether the randomization method is correct; whether allocation concealment is achieved; whether blinding is used; whether outcome data are complete; whether selective reporting is available; and whether other biases are present. Each item will be rated as "low risk", "high risk", or "uncertain". Two reviewers will independently evaluate the risk of bias for inclusion in the study and cross-checked the results.

Strategy of data synthesis: The ratio (OR) will be used for dichotomous data and the weighted mean difference (WMD) for continuous data, or the standardized mean difference (SMD) if the units are inconsistent across studies and could not be converted to agreement. Point estimates and $95 \%$ confidence intervals (Cls) will be calculated for each effect size. Heterogeneity will be judged by Cochran's $Q$ test and 12 values.

Subgroup analysis: According to the subgroup analysis guidelines, we will explore the following subgroup hypotheses in advance to explore the source of heterogeneity: 1. Type of moxibustion: direct moxibustion vs indirect moxibustion; 2. Course of treatment: $\geq 4$ weeks vs $<4$ weeks.

Sensitivity analysis: Excluding studies with high risk of bias and using fixed effects model in meta-analyses.

Language: No restrictions.

Country(ies) involved: China.

Keywords: Moxibustion; chronic obstructive pulmonary disease; protocol; systematic review.
Contributions of each author:

Author 1 - Yanping Wang.

Author 2 - Mingru Huang.

Author 3 - Liping Tang.

Author 4 - Lingxia Xu.

Author 5 - Jiangfeng Wu.

Author 6 - Fei Wang.

Author 7 - Ying Zhang. 